Bull. Chem. Soc. Ethiop. 2021, 35(3), 565-572.

ISSN 1011-3924

(c) 2021 Chemical Society of Ethiopia and The Authors

Printed in Ethiopia

DOI: https://dx.doi.org/10.4314/bcse.v35i3.8

Online ISSN 1726-801X

\title{
NOVEL 6-ARYL-7-ALKYL/ARYL-[1,2,4]TRIAZOLO[4,3-a][1,3,5]TRIAZINE-5(6H)- THIONES, PROCESSES FOR THEIR PREPARATION, CHARACTERIZATION AND EVALUATION OF THEIR IN VITRO ANTIOXIDANT ACTIVITY
}

\author{
Azhar Hajri ${ }^{*}$, Dhouha Alimi, Kaies Rtibi and Hichem Sebai \\ Laboratory of Functional Physiology and Valorization of Bio-resources (UR17ES27), Higher \\ Institute of Biotechnology of Beja, Habib Bourguiba Street, Box 382, 9000 Beja, Jendouba \\ University, Tunisia
}

(Received March 1, 2021; Revised December 23, 2021; Accepted December 24, 2021)

\begin{abstract}
A series of nine new 6-aryl-7-alkyl/aryl-[1,2,4]triazolo[4,3- $a][1,3,5]$ triazine-5(6H)-thiones (2a-i) were synthesized by a reaction of $N$-triazol-3-yl imidates (1) with three different isothiocyanate derivatives (RNCS) in refluxing toluene. The structures of the final heterocyclic compounds were confirmed by ${ }^{1} \mathrm{H}-\mathrm{NMR},{ }^{13} \mathrm{C}-\mathrm{NMR}$, FT-IR, elemental analysis, and mass spectral analysis. The target compounds (2a-i) were in vitro screened for their activity as antioxidants using DPPH (2,2'-diphenyl-1-picrylhydrazyl) and FRAP (ferric reducing/antioxidant power) methods. The results revealed that some triazolotriazine-5- $(6 H)$ thiones exhibited antioxidant activity ranging from moderate to high. The obtained findings revealed that the triazolotriazine-5- $(6 H)$ thiones $(\mathbf{2 g}, \mathbf{2 h}$, and $2 \mathbf{i})$ have superiority among all compounds, It is obvious that the presence of a hydroxyl group in the structure is essential for the antioxidant properties and should be taken into consideration in further design of structures with potential antioxidant properties.
\end{abstract}

KEY WORDS: Imidates, Isothiocyanates, Antioxidant, Triazole, DPPH, FRAP

\section{INTRODUCTION}

Extensive research in the organic-medicinal chemistry field has led to the discovery of different classes of bioactive substances, most of being sulfur and nitrogen-containing heterocycles [1-4]. Heterocyclic compounds are present in various drugs, several natural products, some vitamins, biomolecules, and biologically active compounds such as anti-inflammatory [5], antitumour [6], antimalarial [7], antidepressant [8], anti-HIV [9], and antimicrobial [10] agents. Antioxidants (natural or synthetic) are the molecules, which are able to neutralize free radicals by acting at various stages like interception, prevention and repair [11-13]. It is, therefore, necessary to develop therapeutic agents with improved potential for treating broad spectrum of oxidant infections. In this work, we focused on the design, synthesis, and characterization of new triazolo[4,3-a][1,3,5]triazine-5(6H)-thiones (2a-i). The target heterocyclic compounds (2a-i) were evaluated for their antioxidant activity using different assays.

\section{RESULTS AND DISCUSION}

\section{Chemistry}

The 3-amino[1,2,4]triazole was reacted with orthoesters in the presence of acetic acid to afford the imidates (1) which has been described [14]. The reaction between $N$-triazol-3-yl imidates (1) with appropriate isothiocyanate under reflux of toluene leads to 6-aryl-7-alkyl/aryl$[1,2,4]$ triazolo $[4,3-a][1,3,5]$ triazine-5(6H)-thione (2a-i) (Scheme 1, Table 1).

*Corresponding author. E-mail: lazharhajri.fsb@gmail.com

This work is licensed under the Creative Commons Attribution 4.0 International License 
<smiles>[R1]C(=Nc1nnc[nH]1)OCC</smiles>

(1)

\section{$\underset{\text { Toluène/Refluxing }}{\mathrm{R}^{2}-\mathrm{N}=\mathrm{C}=\mathrm{S}}$}

$\mathbf{R}^{1}: \mathrm{Me}, \mathrm{Et}, \mathrm{Ph}$

$R^{2}: \mathrm{Ph}_{-} \mathrm{CH}_{2}, \mathrm{NO}^{\mathrm{CH}_{2}}$, 我<smiles>[R]c1nc2nncn2c(=S)n1[R]</smiles>

(2a-i)

Scheme 1. Synthesis reaction of 6-aryl-7-alkyl/aryl-[1,2,4]triazolo[4,3-a][1,3,5]triazine-5(6H)thione (2a-i).

Table 1. The results of synthesis of 6-aryl-7-alkyl/aryl-[1,2,4]triazolo[4,3-a][1,3,5]triazine-5(6H)-thione (2ai).

\begin{tabular}{|c|c|c|c|c|c|}
\hline Entry & Products & $\mathrm{R}^{1}$ & $\mathrm{R}^{2}$ & Chemical Formula & Time (hours) \\
\hline 1 & (2a) & $\mathrm{Me}$ & $\mathrm{Ph}-\mathrm{CH}_{2}$ & $\mathrm{C}_{12} \mathrm{H}_{11} \mathrm{~N}_{5} \mathrm{~S}$ & 36 \\
\hline 2 & (2b) & Et & $\mathrm{Ph}-\mathrm{CH}_{2}$ & $\mathrm{C}_{13} \mathrm{H}_{13} \mathrm{~N}_{5} \mathrm{~S}$ & 48 \\
\hline 3 & (2c) & $\mathrm{Ph}$ & $\mathrm{Ph}-\mathrm{CH}_{2}$ & $\mathrm{C}_{17} \mathrm{H}_{13} \mathrm{~N}_{5} \mathrm{~S}$ & 24 \\
\hline 4 & (2d) & $\mathrm{Me}$ & & $\mathrm{C}_{10} \mathrm{H}_{9} \mathrm{~N}_{5} \mathrm{OS}$ & 72 \\
\hline 5 & (2e) & Et & & $\mathrm{C}_{11} \mathrm{H}_{11} \mathrm{~N}_{5} \mathrm{OS}$ & 72 \\
\hline 6 & (2f) & $\mathrm{Ph}$ & & $\mathrm{C}_{15} \mathrm{H}_{11} \mathrm{~N}_{5} \mathrm{OS}$ & 48 \\
\hline 7 & $(2 \mathrm{~g})$ & $\mathrm{Me}$ & & $\mathrm{C}_{19} \mathrm{H}_{25} \mathrm{~N}_{5} \mathrm{OS}$ & 36 \\
\hline 8 & (2h) & Et & & $\mathrm{C}_{20} \mathrm{H}_{27} \mathrm{~N}_{5} \mathrm{OS}$ & 24 \\
\hline 9 & (2i) & $\mathrm{Ph}$ & & $\mathrm{C}_{24} \mathrm{H}_{27} \mathrm{~N}_{5} \mathrm{OS}$ & 24 \\
\hline
\end{tabular}

$\mathrm{Et}=\mathrm{CH}_{3}-\mathrm{CH}_{2}, \mathrm{Me}=\mathrm{CH}_{3}, \mathrm{Ph}=\mathrm{C}_{6} \mathrm{H}_{5}$.

The structure of the products (2a-i) was established with help of the spectral data. The IR spectra of compounds (2a-i) revealed the absorption bands corresponding to $\mathrm{C}=\mathrm{N}$ and $\mathrm{C}=\mathrm{S}$ in the region of 1615-1612 and 1272-1270 $\mathrm{cm}^{-1}$, respectively, and revealed the absence of the absorption band of the NH group. The IR spectra of (2g-i) showed a band at around $3580 \mathrm{~cm}^{-1}$ which was assigned to the new hydroxyl $(\mathrm{OH})$ band in the 2,6-di-tert-butylphenol motif $\left(\mathbf{R}^{2}\right)$. The ${ }^{1} \mathrm{H}$ - NMR spectrum of heterocyclic compounds (2a-i) revealed the disappearance of the signals of $\mathrm{NH}$ and the ethoxy (OEt) groups. The presence of hydroxyl motif in molecules (2g-i) was confirmed by the presence of $\mathrm{D}_{2} \mathrm{O}$-exchangeable signals at $\delta 10.25(\mathbf{2 g}), 10.68(\mathbf{2 h})$, or $10.47 \mathrm{ppm}(\mathbf{2 i})$ assigned to the 2,6-di-tert-butylphenol moiety introduced by isothiocyante further confirmed the cyclization. ${ }^{13} \mathrm{C}-\mathrm{NMR}$ spectra of (2a-i) exhibit a signal at around $\delta 180 \mathrm{ppm}$ corresponding to the carbon of $\mathrm{C}=\mathrm{S}$ motif and display the characteristic signals of all carbons (see experimental part). 


\section{Antioxidant evaluation}

In this paper, the in vitro antioxidant properties of the newly synthesized compounds (2a-i) at different concentrations $(25 ; 50$ and $100 \mu \mathrm{g} / \mathrm{mL}$ ) on DPPH (Figure 1) and FRAP (Figure 2) were examined. It was found from Table 2 that newly synthesized heterocyclic compounds showed various antioxidant activities relative to BHT. In fact, among the analysed structures, highest DPPH radical scavenging activity was demonstrated (2i) compound $\left(\mathrm{IC}_{50}=159 \mu \mathrm{g} / \mathrm{mL}\right)$ followed by $(\mathbf{2 h})\left(\mathrm{IC}_{50}=210 \mu \mathrm{g} / \mathrm{mL}\right)$ and $(\mathbf{2 g})\left(\mathrm{IC}_{50}=252 \mu \mathrm{g} / \mathrm{mL}\right)$. Additionally, compounds (2a-c) showed moderate DPPH radical scavenging activity at all tested concentrations (Table 2). However, no such inhibitory on DPPH were seen with compounds (2d-f). In this study, the obtained $\mathrm{IC}_{50}$ values of all triazolotriazine-5-(6H)thione were lower than that of BHT $\left(\mathrm{IC}_{50}=26.5 \mu \mathrm{g} / \mathrm{mL}\right)$.

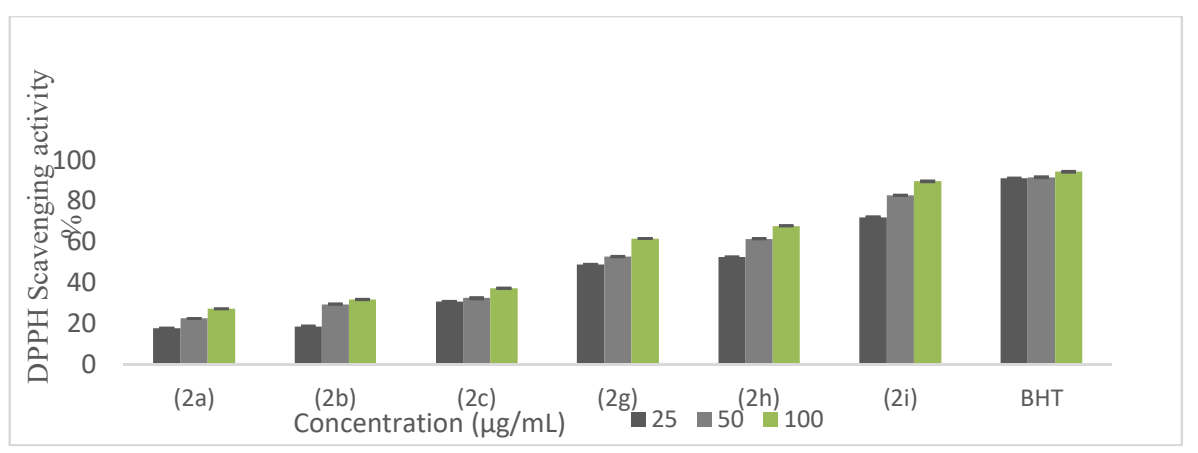

Figure 1. DPPH radical scavenging activity of studied triazolo[4,3- $a][1,3,5]$ triazine-5 $(6 H)$ thiones derivates $(\mathbf{2} \mathbf{a}, \mathbf{2 b}, \mathbf{2 c}, \mathbf{2} \mathbf{g}, \mathbf{2 h}, \mathbf{2 i})$ and BHT. Each value is expressed as mean \pm $\mathrm{SD}, \mathrm{n}=3$. Significant difference was calculated against BHT; $\mathrm{p}<0.0001$.

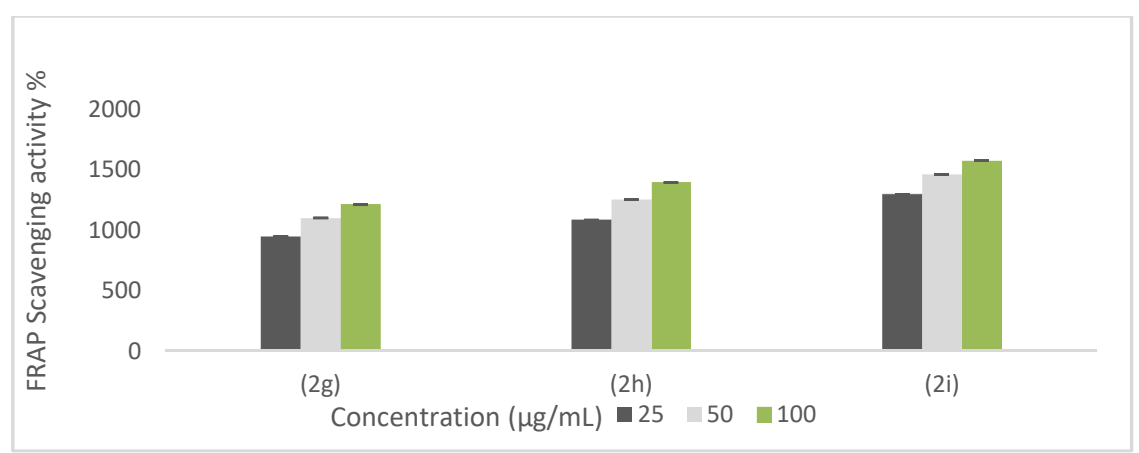

Figure 2. Ferric reducing antioxidant power (FRAP) of studied triazolo[4,3- $a][1,3,5]$ triazine$5(6 H)$-thiones derivates $(\mathbf{2 g}, \mathbf{2} \mathbf{h}, \mathbf{2 i})$. Each value is expressed as mean $\pm \mathrm{SD}, \mathrm{n}=3$.

Table 2. DPPH-radical scavenging of studied compounds (2a-i).

\begin{tabular}{|c|c|}
\hline Compounds & DPPH IC $_{50}(\mu \mathrm{g} / \mathrm{mL})$ \\
\hline $\mathbf{( 2 g})$ & $252 \pm 3.1^{* * *}$ \\
\hline $\mathbf{( 2 h})$ & $210 \pm 2.0^{* * *}$ \\
\hline $\mathbf{( 2 i})$ & $159 \pm 0.2^{* * *}$ \\
\hline BHT & $26.5 \pm 0.3$ \\
\hline
\end{tabular}

Data expressed as mean $\pm \mathrm{SD}, \mathrm{n}=3$; significant difference was calculated against control; $* * * \mathrm{p}<0.0001$.

Bull. Chem. Soc. Ethiop. 2021, 35(3) 

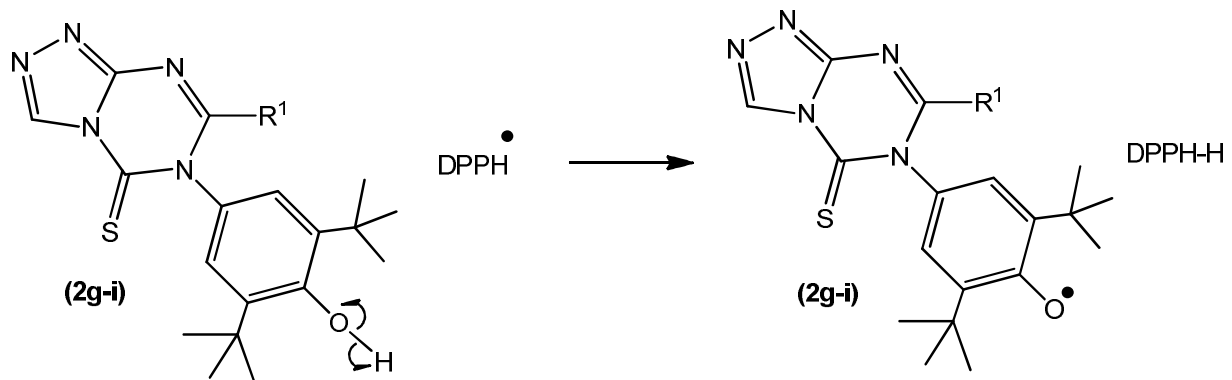

Scheme 2. The probable mechanism for the reaction of compounds (2g-i) with DPPH radical.

In accordance with other reported antioxidant results, the triazolotriazine-5- $(6 H)$ thione is expected to undergo a sequential proton loss electron transfer (SPLET) mechanism as illustrated in Scheme 2.

In the performed FRAP assay, as depicted in Figure 2, among the synthesized compounds only (2g, $\mathbf{2 h}$ and $\mathbf{2 i})$ showed activity in the FRAP method, this result is due to the presence of hydroxyl group manifesting some activity in the FRAP method. While compound (2i) demonstrated the highest activity $(1573 \pm 0.33 \mu \mathrm{mol}$ Trolox/100 g), followed by (2h) (1393 \pm $0.55 \mu \mathrm{mol}$ Trolox/100 g) and (2g) $(1210 \pm 0.33 \mu \mathrm{mol}$ Trolox/100 g), at same concentration close to $100 \mu \mathrm{g} / \mathrm{mL}$. These effects are probably due to the possibility of the analytes breaking up the free radical chain by donating a hydrogen atom (Scheme 3).

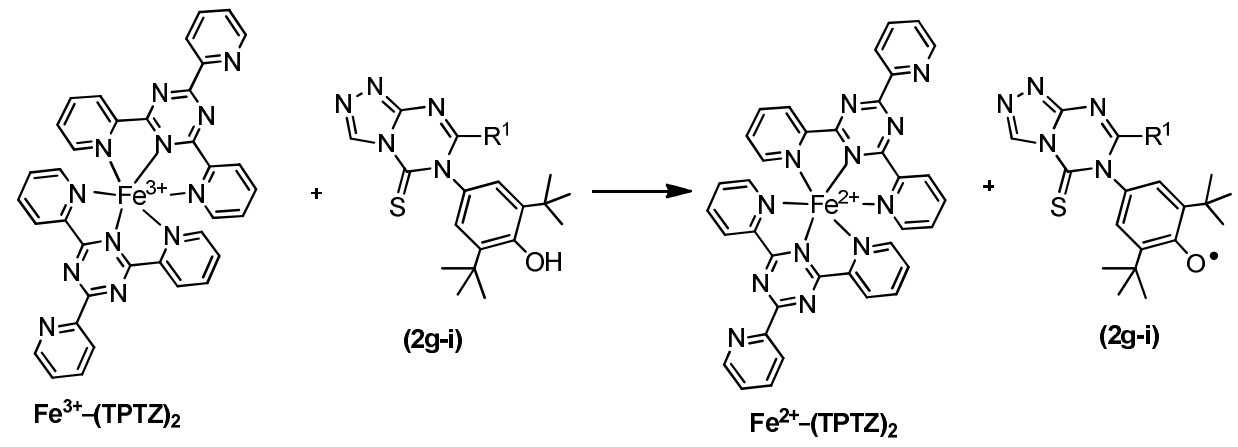

Scheme 3. The probable mechanism for the ferric reducing antioxidant power (FRAP) of compounds (2g-i).

As seen from the presented results for the two discussed methods used for evaluation of the free radicals scavenging activity and FRAP of the newly synthesized structures, the highest antioxidant activity was demonstrated by compound (2i). We believe that this result is due to the presence of 2,6-di-tert-butylphenol group $\left(\mathbf{R}^{2}\right)$ and phenyl ring $\left(\mathbf{R}^{1}\right)$ in the structure of this product.

\section{CONCLUSION}

In this work, a total of nine new 6-aryl-7-alkyl/aryl-[1,2,4]triazolo[4,3- $a][1,3,5]$ triazine-5(6H)thione derivatives (2a-i) were successfully prepared by the reaction of $N$-triazol-3-yl imidates (1) and appropriate isothiocyanate as the reactants. Based on the obtained result, in all two assays used, the (2i) products have been found to possess promising antioxidant activity. Moreover, 
depth study on the free radicals and antioxidants area, to understand their mechanisms and characteristics, should be accelerated as they are of valuable points in preventing different diseases and displayed a favorable treatment approach.

\section{EXPERIMENTAL}

\section{Chemicals}

IR spectra were recorded with a Fourier Transform Infrared Spectrometer (Nicolet IR 200 FT-IR, USA). ${ }^{1} \mathrm{H}$ and ${ }^{13} \mathrm{C}-\mathrm{NMR}$ spectra were recorded with dimethyl sulfoxide- $\mathrm{d}_{6}$ (DMSO- $\mathrm{d}_{6}$ ) solvent containing tetramethylsilane (TMS) on a Bruker 300 spectrometer (USA) $\left({ }^{1} \mathrm{H}: 300 \mathrm{MHz},{ }^{13} \mathrm{C}\right.$ : $75.47 \mathrm{MHz}$ ). The chemical shifts were reported in $\delta$ values relative to TMS (internal reference) for ${ }^{1} \mathrm{H}$ and ${ }^{13} \mathrm{C}$. For the ${ }^{1} \mathrm{H}-\mathrm{NMR}$, the multiplicities of signals are indicated by the following abbreviations: s: singlet, d: doublet, t: triplet, q: quartet, m: multiplet, dd: doublet of doublets. Melting point (mp) was determined on an Electrothermal 9100 melting point apparatus (WeissGallenkamp, Loughborough, UK). Elemental microanalysis was performed on a Perkin-Elmer analyzer apparatus (model 2400, series II-CHN, USA). The electron spray ionization (ESI) positive MS spectra were recorded on a Brüker Daltonics LC-MS spectrometer (USA). All chemicals, DPPH, butylated hydroxyl toluene (BHT), 2,4,6-tripyridyl-s-triazine (TPTZ), potassium ferricyanide, Trolox, ferrous chloride $\left(\mathrm{FeCl}_{2}\right)$, and ferric chloride $\left(\mathrm{FeCl}_{3}\right)$ reagents and solvents were obtained from Sigma-Aldrich Company and were used without any purification. The completion of the reaction was monitored by TLC.

Synthesis of 6-aryl-7-alkyl/aryl-[1,2,4]triazolo[4,3-a][1,3,5]triazine-5(6H)-thione (2a-i). A mixture of $N$-triazol-3-yl imidates (1) $(0.001 \mathrm{~mol})$ and the appropriate isothiocyanate $(0.001 \mathrm{~mol})$ in dry toluene was refluxed for 24-72 hours. The solid material obtained on cooling was filtered off and recrystallized from ethanol.

6-Benzyl-7-methyl-[1,2,4]triazolo[4,3-a][1,3,5]triazine-5(6H)-thione (2a). (102.8 mg, 40\%); a beige solid; mp 182-184 ${ }^{\circ} \mathrm{C}$; IR (FT-IR 200, v $\left(\mathrm{cm}^{-1}\right)$ ): $1272(\mathrm{C}=\mathrm{S}), 1612(\mathrm{C}=\mathrm{N}) ;{ }^{1} \mathrm{H}$ NMR $(300$ MHz, DMSO- $\left.d_{6}\right): \delta 2.48\left(\mathrm{~s}, 3 \mathrm{H}, \mathrm{CH}_{3}-\mathrm{C}(\mathrm{N})=\mathrm{N}\right), 4.71\left(\mathrm{~s}, 2 \mathrm{H}, \mathrm{Ph}-\underline{\mathrm{C}}_{2}-\mathrm{N}\right), 7,32-7,46(\mathrm{~m}, 5 \mathrm{Ar}-\underline{\mathrm{H}})$, $8.89(\mathrm{~s}, 1 \mathrm{H}, \mathrm{N}=\mathrm{CH}-\mathrm{N}-\mathrm{C}=\mathrm{S}) ;{ }^{13} \mathrm{C} \mathrm{NMR}\left(75.47 \mathrm{MHz}, \mathrm{DMSO}-d_{6}\right): \delta 21.9\left(\mathrm{CH}_{3}-\mathrm{C}(\mathrm{N})=\mathrm{N}\right), 50.8(\mathrm{Ph}-$

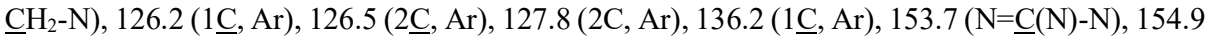
$\left(\mathrm{N}=\underline{\mathrm{C}}\left(\mathrm{CH}_{3}\right)-\mathrm{N}\right), 155.8(\mathrm{~N}=\underline{\mathrm{CH}}-\mathrm{N}), 180.2(\mathrm{~N}-\underline{\mathrm{C}}(\mathrm{N})=\mathrm{S})$, ESI-MS $[\mathrm{M}+\mathrm{H}]^{+}: m / z=258$. Anal. calcd. for $\mathrm{C}_{12} \mathrm{H}_{11} \mathrm{~N}_{5} \mathrm{~S}(\%)$ : C, 56.01; H, 4.31; N, 27.22. Found: C, 55.98; H, 4.32; N, 27.20.

6-Benzyl-7-ethyl-[1,2,4]triazolo[4,3-a][1,3,5]triazine-5(6H)-thione (2b). (122.0 mg, 45\%); a beige solid; mp 190-192 ${ }^{\circ} \mathrm{C}$; IR (FT-IR 200, $\left.v\left(\mathrm{~cm}^{-1}\right)\right)$ : $1270(\mathrm{C}=\mathrm{S}), 1612(\mathrm{C}=\mathrm{N}) ;{ }^{1} \mathrm{H}$ NMR $(300$ $\left.\mathrm{MHz}, \mathrm{DMSO}-d_{6}\right): \delta 1.22\left(\mathrm{t}, 3 \mathrm{H},{ }^{3} J_{H H}=9.0 \mathrm{~Hz}, \mathrm{CH}_{3}-\mathrm{CH}_{2}-\mathrm{C}(\mathrm{N})=\mathrm{N}\right), 2.82\left(\mathrm{q}, 2 \mathrm{H},{ }^{3} J_{H H}=9.0 \mathrm{~Hz}\right.$, $\left.\mathrm{CH}_{3}-\mathrm{CH}_{2}-\mathrm{C}(\mathrm{N})=\mathrm{N}\right), 4.75\left(\mathrm{~s}, 2 \mathrm{H}, \mathrm{Ph}-\mathrm{CH}_{2}-\mathrm{N}\right), 7.32-7.48(\mathrm{~m}, 5 \mathrm{Ar}-\underline{\mathrm{H}}), 8.90(\mathrm{~s}, 1 \mathrm{H}, \mathrm{N}=\mathrm{CH}-\mathrm{N}) ;{ }^{13} \mathrm{C}$ NMR (75.47 MHz, DMSO- $\left.d_{6}\right): \delta 12.3\left(\underline{\mathrm{CH}}_{3}-\mathrm{CH}_{2}-\mathrm{C}(\mathrm{N})=\mathrm{N}\right), 25.8\left(\mathrm{CH}_{3}-\underline{\mathrm{CH}}_{2}-\mathrm{C}(\mathrm{N})=\mathrm{N}\right), 50.1(\mathrm{Ph}-$ $\left.\underline{\mathrm{CH}_{2}}-\mathrm{N}\right), 126.3$ (1드, Ar), $126.6(2 \underline{\mathrm{C}}, \mathrm{Ar}), 127.2(2 \underline{\mathrm{C}}, \mathrm{Ar}), 136.5(1 \underline{\mathrm{C}}, \mathrm{Ar}), 153.8(\mathrm{~N}=\underline{\mathrm{C}}(\mathrm{N})-\mathrm{N}), 154.6$ $\left(\mathrm{N}=\underline{\mathrm{C}}\left(\mathrm{CH}_{3}\right)-\mathrm{N}\right), 155.7(\mathrm{~N}=\underline{\mathrm{CH}}-\mathrm{N}), 180.4(\mathrm{~N}-\underline{\mathrm{C}}(\underline{\mathrm{N}})=\mathrm{S}) ;$ ESI-MS $[\mathrm{M}+\mathrm{H}]^{+}: \mathrm{m} / z=272$. Anal. calcd. for $\mathrm{C}_{13} \mathrm{H}_{13} \mathrm{~N}_{5} \mathrm{~S}$ (\%): C, 57.54; H, 4.83; N, 25.81. Found: C, 57.55; H, 4.85; N, 25.82.

6-Benzyl-7-phenyl-[1,2,4]triazolo[4,3-a][1,3,5]triazine-5(6H)-thione (2c). (178.6 mg, 56\%); a yellowish solid; mp 232-234 ${ }^{\circ} \mathrm{C}$; IR (FT-IR 200, $v\left(\mathrm{~cm}^{-1}\right)$ ): $1271(\mathrm{C}=\mathrm{S}), 1612(\mathrm{C}=\mathrm{N}) ;{ }^{1} \mathrm{H}$ NMR $\left(300 \mathrm{MHz}, \mathrm{DMSO}-d_{6}\right): \delta 4.78\left(\mathrm{~s}, 2 \mathrm{H}, \mathrm{Ph}-\mathrm{CH}_{2}-\mathrm{N}\right), 7.28-7.45(\mathrm{~m}, 10 \mathrm{Ar}-\underline{\mathrm{H}}), 8.85(\mathrm{~s}, 1 \mathrm{H}, \mathrm{N}=\mathrm{CH}-$ $\mathrm{N}-\mathrm{C}=\mathrm{S}) ;{ }^{13} \mathrm{C}$ NMR $\left(75.47 \mathrm{MHz}, \mathrm{DMSO}-d_{6}\right): \delta 50.5\left(\mathrm{Ph}^{-} \underline{\mathrm{C}} \mathrm{H}_{2}-\mathrm{N}\right), 126.1$ ( $\left.\overline{2} \underline{\mathrm{C}}, \mathrm{Ar}\right), 126.8(1 \underline{\mathrm{C}}, \mathrm{Ar})$,

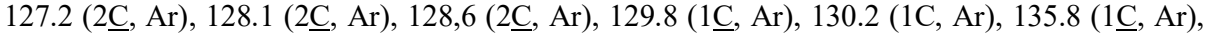
$153.8(\mathrm{~N}=\underline{\mathrm{C}}(\mathrm{N})-\mathrm{N}), 154.7(\mathrm{~N}=\underline{\mathrm{C}}(\mathrm{Ph})-\mathrm{N}), 155.6(\mathrm{~N}=\underline{\mathrm{CH}}-\mathrm{N}), 180.8(\mathrm{~N}-\underline{\mathrm{C}}(\mathrm{N})=\mathrm{S}) ;$ ESI-MS $[\mathrm{M}+\mathrm{H}]^{+}$: 
$m / z=320$. Anal. calcd. for $\mathrm{C}_{17} \mathrm{H}_{13} \mathrm{~N}_{5} \mathrm{~S}(\%)$ : C, 63.93; $\mathrm{H}, 4.10 ; \mathrm{N}, 21.93$. Found: $\mathrm{C}, 63.91 ; \mathrm{H}, 4.12$; $\mathrm{N}, 21.95$.

6-(Furan-2-ylmethyl)-7-methyl-[1,2,4]triazolo[4,3-a][1,3,5]triazine-5(6H)-thione (2d). (126.11 $\mathrm{mg}, 51 \%$ ); a yellowish needles solid; $\mathrm{mp} 221-223^{\circ} \mathrm{C}$; IR (FT-IR 200, $v\left(\mathrm{~cm}^{-1}\right)$ ): $1270(\mathrm{C}=\mathrm{S}), 1614$ $(\mathrm{C}=\mathrm{N}) ;{ }^{1} \mathrm{H}$ NMR $\left(300 \mathrm{MHz}, \mathrm{DMSO}-d_{6}\right): \delta 2.64\left(\mathrm{~s}, 3 \mathrm{H}, \mathrm{CH}_{3}-\mathrm{C}(\mathrm{N})=\mathrm{N}\right), 3.94(\mathrm{~s}, 2 \mathrm{H}, \mathrm{CH}=\mathrm{C}(\mathrm{O})-$ $\left.\mathrm{C}_{2}-\mathrm{N}\right), 6.78\left(\mathrm{dd}, 1 \mathrm{H},{ }^{3} J_{H H}=3.6 \mathrm{~Hz},{ }^{3} J_{H H}=1.64 \mathrm{~Hz}, \mathrm{CH}=\mathrm{C} \underline{H}-\mathrm{CH}=\mathrm{C}(\mathrm{O})-\mathrm{CH}_{2}-\mathrm{N}\right), 7.61(\mathrm{~d}, 1 \mathrm{H}$, $\left.{ }^{3} J_{H H}=3.4 \mathrm{~Hz}, \mathrm{C} \underline{\mathrm{H}}=\mathrm{C}(\mathrm{O})-\mathrm{CH}_{2}-\mathrm{N}\right), 8.05\left(\mathrm{~d}, 1 \mathrm{H},{ }^{3} \mathrm{~J}_{\mathrm{HH}}=1.64 \mathrm{~Hz}, \mathrm{C} \underline{\mathrm{H}}=\mathrm{CH}-\mathrm{CH}=\mathrm{C}(\mathrm{O})-\mathrm{CH}_{2}-\mathrm{N}\right), 8.80$ $(\mathrm{s}, 1 \mathrm{H}, \mathrm{N}=\mathrm{CH}-\mathrm{N}-\mathrm{C}=\mathrm{S}) ;{ }^{13} \mathrm{C}$ NMR $\left(75.47 \mathrm{MHz}, \mathrm{DMSO}-d_{6}\right): \delta 20.8\left(\mathrm{CH}_{3}-\mathrm{C}(\mathrm{N})=\mathrm{N}\right), 52.3$ $\left(\mathrm{CH}=\mathrm{C}(\mathrm{O})-\underline{\mathrm{CH}}_{2}-\mathrm{N}\right), \quad 111.2\left(\mathrm{CH}=\mathrm{CH}-\underline{\mathrm{CH}}=\mathrm{C}(\mathrm{O})-\mathrm{CH}_{2}-\mathrm{N}\right), 111.8 \quad\left(\mathrm{CH}=\underline{\mathrm{CH}}-\mathrm{CH}=\mathrm{C}(\mathrm{O})-\mathrm{CH}_{2}-\mathrm{N}\right)$, $143.6\left(\underline{\mathrm{CH}}=\mathrm{CH}-\mathrm{CH}=\mathrm{C}(\mathrm{O})-\mathrm{CH}_{2}-\mathrm{N}\right), 149.5 \quad\left(\mathrm{CH}=\mathrm{CH}-\mathrm{CH}=\underline{\mathrm{C}}(\mathrm{O})-\mathrm{CH}_{2}-\mathrm{N}\right), 153.8 \quad(\mathrm{~N}=\underline{\mathrm{C}}(\mathrm{N})-\mathrm{N})$, $154.2\left(\mathrm{~N}=\underline{\mathrm{C}}\left(\mathrm{CH}_{3}\right)-\mathrm{N}\right), 155.6(\mathrm{~N}=\mathrm{CH}-\mathrm{N}), 180.4(\mathrm{~N}-\underline{\mathrm{C}}(\mathrm{N})=\mathrm{S}) ;$ ESI-MS $[\mathrm{M}+\mathrm{H}]^{+}: m / z=248$. Anal. calcd. for $\mathrm{C}_{10} \mathrm{H}_{9} \mathrm{~N}_{5} \mathrm{OS}(\%)$ : C, 48.57; H, 3.67; N, 28.32. Found: C, 48.58; H, 3.69; N, 28.30.

7-Ethyl-6-(furan-2-ylmethyl)-[1,2,4]triazolo[4,3-a][1,3,5]triazine-5(6H)-thione (2e). (112.35 mg, 43\%); a dark yellow solid; mp: $261-263{ }^{\circ} \mathrm{C}$; IR (FT-IR 200, $\left.v\left(\mathrm{~cm}^{-1}\right)\right): 1272(\mathrm{C}=\mathrm{S}), 1615$ $(\mathrm{C}=\mathrm{N}) ;{ }^{1} \mathrm{H}$ NMR $\left(300 \mathrm{MHz}, \mathrm{DMSO}-d_{6}\right): \delta 1.25\left(\mathrm{t}, 3 \mathrm{H},{ }^{3} J_{\mathrm{HH}}=8.7 \mathrm{~Hz}, \mathrm{CH}_{3}-\mathrm{CH}_{2}-\mathrm{C}(\mathrm{N})=\mathrm{N}\right), 2.78$ $\left(\mathrm{q}, 2 \mathrm{H},{ }^{3} J_{H H}=9.0 \mathrm{~Hz}, \mathrm{CH}_{3}-\mathrm{CH}_{2}-\mathrm{C}(\mathrm{N})=\mathrm{N}\right), 3.92\left(\mathrm{~s}, 2 \mathrm{H}, \mathrm{CH}=\mathrm{C}(\mathrm{O})-\mathrm{C}_{2}-\mathrm{N}\right), 6.76\left(\mathrm{dd}, 1 \mathrm{H},{ }^{3} J_{H H}=\right.$ $\left.3.6 \mathrm{~Hz},{ }^{3} J_{H H}=1.64 \mathrm{~Hz}, \mathrm{CH}=\underline{\mathrm{C}}-\mathrm{CH}=\mathrm{C}(\mathrm{O})-\mathrm{CH}_{2}-\mathrm{N}\right), 7.65\left(\mathrm{~d}, 1 \mathrm{H},{ }^{3} J_{H H}=3.4 \mathrm{~Hz}, \mathrm{CH}=\mathrm{C}(\mathrm{O})-\mathrm{CH}_{2}-\right.$ $\mathrm{N}), 8.08\left(\mathrm{~d}, 1 \mathrm{H},{ }^{3} J_{H H}=1.66 \mathrm{~Hz}, \mathrm{CH}=\mathrm{CH}-\mathrm{CH}=\mathrm{C}(\mathrm{O})-\mathrm{CH}_{2}-\mathrm{N}\right), 8.83(\mathrm{~s}, 1 \mathrm{H}, \mathrm{N}=\mathrm{C} \underline{\mathrm{H}}-\mathrm{N}-\mathrm{C}=\mathrm{S}) ;{ }^{13} \mathrm{C}$ NMR (75.47 MHz, DMSO-d $\left.d_{6}\right) \delta 11.8\left(\underline{\mathrm{CH}}_{3}-\mathrm{CH}_{2}-\mathrm{C}(\mathrm{N})=\mathrm{N}\right), 26.1\left(\mathrm{CH}_{3}-\underline{\mathrm{CH}}_{2}-\overline{\mathrm{C}}(\mathrm{N})=\mathrm{N}\right), 52.8$ $\left(\mathrm{CH}=\mathrm{C}(\mathrm{O})-\underline{\mathrm{CH}}_{2}-\mathrm{N}\right), \quad 112.2 \quad\left(\mathrm{CH}=\mathrm{CH}-\underline{\mathrm{CH}}=\mathrm{C}(\mathrm{O})-\mathrm{CH}_{2}-\mathrm{N}\right), 112.5 \quad\left(\mathrm{CH}=\mathrm{CH}-\mathrm{CH}=\mathrm{C}(\mathrm{O})-\mathrm{CH}_{2}-\mathrm{N}\right)$, $141.9\left(\mathrm{CH}=\mathrm{CH}-\mathrm{CH}=\mathrm{C}(\mathrm{O})-\mathrm{CH}_{2}-\mathrm{N}\right), 149.4 \quad\left(\mathrm{CH}=\mathrm{CH}-\mathrm{CH}=\mathrm{C}(\mathrm{O})-\mathrm{CH}_{2}-\mathrm{N}\right), 153.4 \quad(\mathrm{~N}=\mathrm{C}(\mathrm{N})-\mathrm{N})$, $154.3\left(\mathrm{~N}=\underline{\mathrm{C}}\left(\mathrm{CH}_{3}\right)-\mathrm{N}\right), 155.7(\mathrm{~N}=\underline{\mathrm{CH}}-\mathrm{N}), 180.6(\mathrm{~N}-\mathrm{C}(\mathrm{N})=\mathrm{S}) ; \mathrm{ESI}-\mathrm{MS}[\mathrm{M}+\mathrm{H}]^{+}: m / z=262$. Anal. calcd. for $\mathrm{C}_{11} \mathrm{H}_{11} \mathrm{~N}_{5} \mathrm{OS}(\%)$ : C, 50.56; H, 4.24; N, 26.80. Found: C, 50.58; H, 4.25; N, 26.81 .

6-(Furan-2-ylmethyl)-7-phenyl-[1,2,4]triazolo[4,3-a][1,3,5]triazine-5(6H)-thione (2f). (179.22 mg, 58\%); a dark yellow solid; mp: $205-207{ }^{\circ} \mathrm{C}$; IR (FT-IR 200, $\left.v\left(\mathrm{~cm}^{-1}\right)\right): 1270(\mathrm{C}=\mathrm{S}), 1612$ $(\mathrm{C}=\mathrm{N}) ;{ }^{1} \mathrm{H}$ NMR $\left(300 \mathrm{MHz}, \mathrm{DMSO}-d_{6}\right): \delta 3.96\left(\mathrm{~s}, 2 \mathrm{H}, \mathrm{CH}=\mathrm{C}(\mathrm{O})-\mathrm{C}_{2}-\mathrm{N}\right), 6.72\left(\mathrm{dd}, 1 \mathrm{H},{ }^{3} J_{H H}=\right.$ $\left.3.6 \mathrm{~Hz},{ }^{3} J_{H H}=1.66 \mathrm{~Hz}, \mathrm{CH}=\mathrm{C} \underline{\mathrm{H}}-\mathrm{CH}=\mathrm{C}(\mathrm{O})-\mathrm{CH}_{2}-\mathrm{N}\right), 7.30-7.46(\mathrm{~m}, 5 \mathrm{Ar}-\underline{\mathrm{H}}), 7.63\left(\mathrm{~d}, 1 \mathrm{H},{ }^{3} J_{H H}=3.5\right.$ $\left.\mathrm{Hz}, \mathrm{C} \underline{\mathrm{H}}=\mathrm{C}(\mathrm{O})-\mathrm{CH}_{2}-\mathrm{N}\right), 8.09\left(\mathrm{~d}, 1 \mathrm{H},{ }^{3} J_{H H}=1.66 \mathrm{~Hz}, \quad \mathrm{C} \underline{\mathrm{H}}=\mathrm{CH}-\mathrm{CH}=\mathrm{C}(\mathrm{O})-\mathrm{CH}_{2}-\mathrm{N}\right), 8.83(\mathrm{~s}, 1 \mathrm{H}$, $\mathrm{N}=\mathrm{C} \underline{\mathrm{H}}-\mathrm{N}-\mathrm{C}=\mathrm{S}) ;{ }^{13} \mathrm{C} \mathrm{NMR}\left(75.47 \mathrm{MHz}, \mathrm{DMSO}-d_{6}\right): \delta 20.8\left(\mathrm{CH}_{3}-\mathrm{C}(\mathrm{N})=\mathrm{N}\right), 52.3\left(\mathrm{CH}=\mathrm{C}(\mathrm{O})-\underline{\mathrm{CH}}_{2}-\right.$ $\mathrm{N}), 112.2\left(\mathrm{CH}=\mathrm{CH}-\underline{\mathrm{CH}}=\mathrm{C}(\mathrm{O})-\mathrm{CH}_{2}-\mathrm{N}\right), 112.7\left(\mathrm{CH}=\underline{\mathrm{CH}}-\mathrm{CH}=\mathrm{C}(\mathrm{O})-\mathrm{CH}_{2}-\mathrm{N}\right), 128.3(1 \mathrm{C}, \mathrm{Ar}), 129.3$

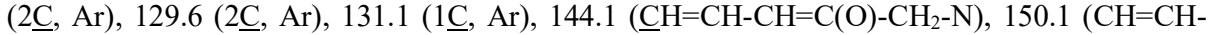
$\left.\mathrm{CH}=\underline{\mathrm{C}}(\mathrm{O})-\mathrm{CH}_{2}-\mathrm{N}\right), 153.1(\mathrm{~N}=\underline{\mathrm{C}}(\mathrm{N})-\mathrm{N}), 154.0\left(\mathrm{~N}=\underline{\mathrm{C}}\left(\mathrm{CH}_{3}\right)-\mathrm{N}\right), 155.7(\mathrm{~N}=\underline{\mathrm{C}} \mathrm{H}-\mathrm{N}), 180.9(\mathrm{~N}-$ $\underline{\mathrm{C}}(\mathrm{N})=\mathrm{S})$; ESI-MS $[\mathrm{M}+\mathrm{H}]^{+}: m / z=310$. Anal. calcd. for $\mathrm{C}_{15} \mathrm{H}_{11} \mathrm{~N}_{5} \mathrm{OS}(\%): \mathrm{C}, 58.24 ; \mathrm{H}, 3.58 ; \mathrm{N}$, 22.64. Found: C, 58.26; H, 3.59; N, 22.62.

6-(3,5-Di-tert-butyl-4-hydroxyphenyl)-7-methyl-[1,2,4]triazolo[4,3-a] [1,3,5] triazine-5(6H)-thione (2g). (192.4 mg, 52\%); a Yellowish needles solid; mp: 283-285 ${ }^{\circ} \mathrm{C}$; IR (FT-IR 200, $v\left(\mathrm{~cm}^{-1}\right)$ ) : $1270(\mathrm{C}=\mathrm{S}), 1612(\mathrm{C}=\mathrm{N}), 3580(\mathrm{OH})$; ${ }^{1} \mathrm{H}$ NMR (300 MHz, DMSO- $\left.d_{6}\right): \delta 1.36(\mathrm{~s}, 18 \mathrm{H}, 2-$ $\left.\mathrm{C}\left(\mathrm{CH}_{3}\right)_{3}\right), 2.51\left(\mathrm{~s}, 3 \mathrm{H}, \mathrm{C}_{3}-\mathrm{C}(\mathrm{N})=\mathrm{N}\right), 7.24(\mathrm{~s}, 2 \mathrm{H}, \mathrm{Ar}-\underline{\mathrm{H}}), 8.86$ (s, 1H, N=CH$\left.-\mathrm{N}-\mathrm{C}=\mathrm{S}\right), 10.25$ (br.s, $1 \mathrm{H}, \mathrm{Ar}-\mathrm{OH}, \mathrm{D}_{2} \mathrm{O}$ exchangeable); ${ }^{13} \mathrm{C}$ NMR $\left(75.47 \mathrm{MHz}, \mathrm{DMSO}-d_{6}\right): \delta 22.8\left(\mathrm{CH}_{3}-\mathrm{C}(\mathrm{N})=\mathrm{N}\right), 31.2$ $\left(6 \mathrm{C},-\mathrm{C}_{A r}-\mathrm{C}\left(\underline{\mathrm{CH}}_{3}\right)_{3}\right), 34.8\left(2 \mathrm{C},-\mathrm{C}_{A r}-\underline{\mathrm{C}}\left(\mathrm{CH}_{3}\right)_{3}\right), 122.2(2 \underline{\mathrm{C}}, \mathrm{Ar}), 126.4\left(-\mathrm{N}-\underline{\mathrm{C}}_{\mathrm{Ar}}\right), 138.1\left(2 \mathrm{C},-\underline{\mathrm{C}}_{A r}-\right.$ $\left.\mathrm{C}\left(\mathrm{CH}_{3}\right)_{3}\right), 150.4\left(1 \underline{\mathrm{C}}_{A r}-\mathrm{OH}\right), 153.1(\mathrm{~N}=\underline{\mathrm{C}}(\mathrm{N})-\mathrm{N}), 154.6\left(\mathrm{~N}=\underline{\mathrm{C}}\left(\mathrm{CH}_{3}\right)-\mathrm{N}\right), 155.7(\mathrm{~N}=\underline{\mathrm{CH}}-\mathrm{N}), 180.9$ $(\mathrm{N}-\mathrm{C}(\mathrm{N})=\mathrm{S})$; ESI-MS $[\mathrm{M}+\mathrm{H}]^{+}: \mathrm{m} / z=372$. Anal. calcd. for $\mathrm{C}_{19} \mathrm{H}_{25} \mathrm{~N}_{5} \mathrm{OS}(\%)$ :, $61.43 ; \mathrm{H}, 6.78$; N, 18.85. Found: C, 61.45; H, 6.79; N, 18.83 .

6-(3,5-Di-tert-butyl-4-hydroxyphenyl)-7-ethyl-[1,2,4]triazolo[4,3-a][1,3,5]triazine-5(6H)-thione (2h). (205.2 mg, 54\%); a yellowish needles solid; mp 270-272 ${ }^{\circ} \mathrm{C}$; IR (FT-IR 200, $v\left(\mathrm{~cm}^{-1}\right)$ ): $1270(\mathrm{C}=\mathrm{S}), 1612(\mathrm{C}=\mathrm{N}), 3580(\mathrm{OH}) ;{ }^{1} \mathrm{H}$ NMR $\left(300 \mathrm{MHz}, \mathrm{DMSO}-d_{6}\right): \delta 1.23\left(\mathrm{t}, 3 \mathrm{H},{ }^{3} J_{H H}=8.7\right.$ 
$\left.\mathrm{Hz}, \mathrm{CH}_{3}-\mathrm{CH}_{2}-\mathrm{C}(\mathrm{N})=\mathrm{N}\right), 1.37\left(\mathrm{~s}, 18 \mathrm{H}, 2-\mathrm{C}\left(\mathrm{CH}_{3}\right)_{3}\right), 2.62\left(\mathrm{q}, 3 \mathrm{H},{ }^{3} \mathrm{~J}_{\mathrm{HH}}=8.9 \mathrm{~Hz}, \mathrm{CH}_{3}-\mathrm{CH}_{2}-\mathrm{C}(\mathrm{N})=\mathrm{N}\right)$, $7,23$ (s, 2H, Ar- $\underline{\mathrm{H}}$ ), 8.86 (s, $1 \mathrm{H}, \mathrm{N}=\mathrm{C} \underline{\mathrm{H}}-\mathrm{N}-\mathrm{C}=\mathrm{S}), 10.68$ (br.s, $1 \mathrm{H}, \mathrm{Ar}-\mathrm{O} \underline{\mathrm{H}}, \mathrm{D}_{2} \mathrm{O}$ exchangeable); ${ }^{13} \mathrm{C}$ NMR (75.47 MHz, DMSO- $\left.d_{6}\right): \delta 11.8\left(\mathrm{CH}_{3}-\mathrm{CH}_{2}-\mathrm{C}(\mathrm{N})=\mathrm{N}\right), 26.1\left(\mathrm{CH}_{3}-\mathrm{CH}_{2}-\mathrm{C}(\mathrm{N})=\mathrm{N}\right), 31.1(6 \mathrm{C}$, $\left.-\mathrm{C}_{A r}-\mathrm{C}\left(\underline{\mathrm{CH}}_{3}\right)_{3}\right), 34.5\left(2 \mathrm{C},-\mathrm{C}_{A r}-\underline{\mathrm{C}}\left(\mathrm{CH}_{3}\right)_{3}\right), 121.8(2 \underline{\mathrm{C}}, \mathrm{Ar}), 153.1(\mathrm{~N}=\underline{\mathrm{C}}(\mathrm{N})-\overline{\mathrm{N}}), 126.2\left(-\mathrm{N}-\underline{\mathrm{C}}_{\mathrm{Ar}}\right), 138.3$ $\left(2 \mathrm{C},-\underline{\mathrm{C}}_{A r}-\mathrm{C}\left(\mathrm{CH}_{3}\right)_{3}\right), 150.2\left(1 \underline{\mathrm{C}}_{A r}-\mathrm{OH}\right), 154.7\left(\mathrm{~N}=\underline{\mathrm{C}}\left(\mathrm{CH}_{3}\right)-\mathrm{N}\right), 155.4(\mathrm{~N}=\underline{\mathrm{C}} \mathrm{H}-\mathrm{N}), 180.8(\mathrm{~N}-$ $\underline{\mathrm{C}}(\mathrm{N})=\mathrm{S})$; ESI-MS $[\mathrm{M}+\mathrm{H}]^{+}: m / z=386$. Anal. calcd. for $\mathrm{C}_{20} \mathrm{H}_{27} \mathrm{~N}_{5} \mathrm{OS}(\%): \mathrm{C}, 62.31 ; \mathrm{H}, 7.06 ; \mathrm{N}$, 18.17. Found: C, 62.32; H, 7.04; N, 18.18 .

6-(3,5-Di-tert-butyl-4-hydroxyphenyl)-7-phenyl-[1,2,4]triazolo[4,3-a][1,3,5]triazine-5(6H)-thione (2i). (219.3 mg, 51\%); a dark Yellow solid; mp 284-286 ${ }^{\circ} \mathrm{C}$; IR (FT-IR 200, $v\left(\mathrm{~cm}^{-1}\right)$ ): 1271(C=S), $1613(\mathrm{C}=\mathrm{N}), 3581(\mathrm{OH}) ;{ }^{1} \mathrm{H}$ NMR $\left(300 \mathrm{MHz}, \mathrm{DMSO}-d_{6}\right): 1.39\left(\mathrm{~s}, 18 \mathrm{H}, 2-\mathrm{C}\left(\mathrm{CH}_{3}\right)_{3}\right)$, 7.23 (s, 2H, Ar- $\underline{\mathrm{H}}$, ), 7.42-7.78 (m, $5 \mathrm{H}, \mathrm{Ar}-\underline{\mathrm{H}}), 8.85$ (s, $1 \mathrm{H}, \mathrm{N}=\mathrm{CH}-\mathrm{N}-\mathrm{C}=\mathrm{S}$ ), 10.47 (br.s, $1 \mathrm{H}, \mathrm{Ar}-$ $\mathrm{OH}, \mathrm{D}_{2} \mathrm{O}$ exchangeable); ${ }^{13} \mathrm{C}$ NMR $\left(75.47 \mathrm{MHz}, \mathrm{DMSO}-d_{6}\right): \delta 31.2\left(6 \mathrm{C},-\mathrm{C}_{A r}-\mathrm{C}\left(\underline{\mathrm{CH}}_{3}\right)_{3}\right), 34.3(2 \mathrm{C}$,

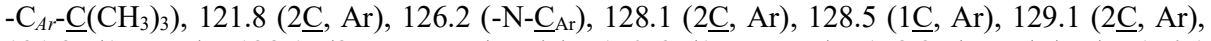
$131.2(1 \underline{\mathrm{C}}, \mathrm{Ar}), 138.1\left(2 \mathrm{C},-\underline{\mathrm{C}}_{A r}-\mathrm{C}\left(\mathrm{CH}_{3}\right)_{3}\right), 150.6\left(1 \underline{\mathrm{C}}_{A r}-\mathrm{OH}\right), 152.9(\mathrm{~N}=\underline{\mathrm{C}}(\mathrm{N})-\mathrm{N}), 154.1$ $\left(\mathrm{N}=\underline{\mathrm{C}}\left(\mathrm{CH}_{3}\right)-\mathrm{N}\right), 155.2(\mathrm{~N}=\underline{\mathrm{CH}}-\mathrm{N}), 180.6(\mathrm{~N}-\underline{\mathrm{C}}(\mathrm{N})=\mathrm{S}) ; \mathrm{ESI}-\mathrm{MS}[\mathrm{M}+\mathrm{H}]^{+}: \mathrm{m} / z=434$. Anal. calcd. for $\mathrm{C}_{24} \mathrm{H}_{27} \mathrm{~N}_{5} \mathrm{OS}(\%)$ : C, 66.49; H, 6.28; N, 16.15. Found: $\mathrm{C}, 66.50 ; \mathrm{H}, 6.29 ; \mathrm{N}, 16.17$.

\section{Antioxidant evaluation}

DPPH radical scavenging activity. The antioxidant activity of triazolotriazine-5- $(6 H)$ thiones (2ai) was performed using DPPH free radical scavenging [15]. The prepared methanol solution of DPPH $(20 \mu \mathrm{g} / \mathrm{mL})$ was stored at $10^{\circ} \mathrm{C}$ in the dark. The heterocyclic compounds (2a-i) were dissolved in methanol. $(0.5 \mathrm{~mL})$ of different concentrations $(25 ; 50 ; 100 \mu \mathrm{g} / \mathrm{mL})$ of the tested compounds was added to DPPH solution $(1.0 \mathrm{~mL})$. Then, the plate was incubated in dark for 30 min at room temperature. At a wavelength of $517 \mathrm{~nm}$, the process of occurrence of discoloration was recorded $5 \mathrm{~min}$ after the reaction and was compared with a blank control. Butylated hydroxyl toluene (BHT) was taken as standard. The free radical scavenging ability of tested compounds expressed as \%inhibition was calculated using the following equation [16]:

$$
\% \mathrm{I}=\left[\left(\mathrm{A}_{\mathrm{c}}-\mathrm{A}_{\mathrm{S}} / \mathrm{Ac}\right)\right] \times 100
$$

where $A_{c}$ means absorbance of control; $A_{s}$ means absorbance of the sample.

Ferric reducing antioxidant power assay (FRAP). Benzie and Strain method with some modifications were conducted in the ferric reducing antioxidant power (FRAP) assay [17]. The stock solutions of $300 \mathrm{mM}$ of the acetate buffer, $\mathrm{pH} 3.6 ; 20 \mathrm{mM} \mathrm{FeCl}_{3} \cdot 6 \mathrm{H}_{2} \mathrm{O}$ solution and $10 \mathrm{mM}$ TPTZ solution in $40-\mathrm{mM} \mathrm{HCl}$ were previously prepared. Three reagents were prepared; acetate buffer $(300 \mathrm{mM}, \mathrm{pH}=3.6), 20 \mathrm{mM} \mathrm{FeCl}_{3} \cdot 6 \mathrm{H}_{2} \mathrm{O}$ solution and $10 \mathrm{mM} \mathrm{TPTZ}$ in $40 \mathrm{mM} \mathrm{HCl}$. The freshly mixed solution was prepared by mixing $\mathrm{FeCl}_{3} \cdot 6 \mathrm{H}_{2} \mathrm{O}$, acetate buffer, and TPTZ in the ratio of 2.5: $25: 2.5(\mathrm{v} / \mathrm{v} / \mathrm{v})$, respectively. The mixture was warmed at $37^{\circ} \mathrm{C}$. In a dark condition, FRAP solution $(2850 \mu \mathrm{L})$ was allowed to react with triazolotriazine-5- $(6 H)$ thiones $(\mathbf{2 a - i})(150 \mu \mathrm{L})$ for $30 \mathrm{~min}$. . Measurement of the absorbance readings of the colored product (ferrous tripyridyltriazine complex) at $593 \mathrm{~nm}$. The results are represented in $\mu \mathrm{mol}$ Trolox/100 g dry matter. When the measured FRAP value exceeded the linear range of the standard curve, an extra additional dilution was applied to lower the measurement with consideration of the dilution factor.

\section{Statistical analysis}

Statview v.5.0.1 software (SAS Institute, Cary, NC) was used for all statistical analyses. Experimental data were presented as mean standard deviation. One-way analysis of variance was used to determine the statistical significance. Differences were considered statistically significant if $\mathrm{p}<0.0001$. 


\section{REFERENCES}

1. Semren, T.Ž.; Žunec, S.; Pizent, A. Oxidative stress in triazine pesticide toxicity: A review of the main biomarker findings. Arh. Hig. Rada Toksikol. 2018, 69, 109-125.

2. Abuelizz, H.A.; Taie, H.A.A.; Marzouk, M.; Al-Salahi, R. Synthesis and antioxidant activity of 2-methylthio-pyrido[3,2-e][1,2,4] triazolo[1,5-a]pyrimidines. Open Chem. 2019, 17, 823830.

3. Um, S.; Lee, J.; Kang, Y.; Baek, D. The synthesis and properties of triazine-stilbene fluorescent brighteners containing the phenolic antioxidant. Dyes Pigm. 2005, 64, 93-99.

4. Gaidhane, M.K. Antioxidant, Antifungal Activity and Isomerisation: Synthesis of 1,2,3,5Thiatriazines in: Proceedings of the ICSESD-2017; Infogain Publication, 2017; pp. 163-171.

5. Ahmadi, F.; Ghayahbashi, M.; Sharifzadeh, M.; Alipoiur, E.; Ostad, S.; Vosooghi, M.; khademi, H.; Amini, M. Synthesis and evaluation of anti-inflammatory and analgesic activities of new 1,2,4-triazole derivatives. Med. Chem. 2014, 11, 69-76.

6. Zhang, Y.; Liu, X.; Lin, Y.; Lian, B.; Lan, W.; Iovanna, J.L.; Liu, X.; Peng, L.; Xia, Y. Novel triazole nucleoside analogues promote anticancer activity via both apoptosis and autophagy. Chem. Commun. 2020, 56, 10014-10017.

7. Mabasa, T.F.; Awe, B.; Laming, D.; Kinfe, H.H. Design, synthesis and antiplasmodial evaluation of sulfoximine-triazole hybrids as potential antimalarial prototypes. Med. Chem. 2019, 15, 685-692.

8. Song, M.-X.; Huang, Y.-S.; Zhou, Q.-G.; Deng, X.-Q.; Yao, X.-D. Synthesis of ring-opened derivatives of triazole-containing quinolinones and their antidepressant and anticonvulsant activities. Bioorg. Chem. 2021, 106, 104505.

9. Hilimire, T.A.; Chamberlain, J.M.; Anokhina, V.; Bennett, R.P.; Swart, O.; Myers, J.R.; Ashton, J.M.; Stewart, R.A.; Featherston, A.L.; Gates, K.; Helms, E.D.; Smith, H.C.; Dewhurst, S.; Miller, B.L. HIV-1 frameshift RNA-targeted triazoles inhibit propagation of replication-competent and multi-drug-resistant HIV in human cells. ACS Chem. Biol. 2017, $12,1674-1682$.

10. Lin, L.; Liu, H.; J. Wang, D.; J. Hu, Y.; H. Wei, X. Synthesis and biological activities of 3,6disubstituted-1,2,4-triazolo-1,3,4- thiadiazole derivatives. Bull. Chem. Soc. Ethiop. 2018, 31, 481-489.

11. Nakbi, H.; Dallel, W.; Hammami, S.; Mighri, Z. Phytochemical profile and antioxidant properties of leaves extracts from Posidonia oceanica (L.) Delile and their allelopathic potential on terrestrial plant species. Bull. Chem. Soc. Ethiop. 2020, 34, 437-447.

12 Naima, B.; Abdelkrim, R.; Ouarda, B.; Salah, N.N.; Larbi, B. a. M. Chemical composition, antimicrobial, antioxidant and anticancer activities of essential oil from Ammodaucus leucotrichus Cosson \& Durieu (Apiaceae) growing in south Algeria. Bull. Chem. Soc. Ethiop. 2019, 33, 541-549.

13. Hirano, R.; Sasamoto, W.; Matsumoto, A.; Itakura, H.; Igarashi, O.; Kondo, K. Antioxidant ability of various flavonoids against DPPH radicals and LDL oxidation. J. Nutr. Sci. Vitaminol. 2001, 47, 357-362.

14. Abderrahim, R. A Novel Synthetic Route to New 1,2,4-Triazolo-1,3,5-triazin-4-ones derivatives. Phosphorus, Sulfur Silicon Relat. Elem. 2006, 181, 581-585.

15. Brand-Williams, W.; Cuvelier, M.E.; Berset, C. Use of a free radical method to evaluate antioxidant activity. LWT - Food Sci. Technol. 1995, 28, 25-30.

16. San Miguel-Chávez, R. Phenolic Antioxidant Capacity: A Review of the State of the Art. in: Phenolic Compounds - Biological Activity; Soto-Hernndez, M.; Palma-Tenango, M.; GarciaMateos, M. del R., Eds.; InTech; 2017.

17. Benzie, I.F.F.; Strain, J.J. The ferric reducing ability of plasma (FRAP) as a measure of "antioxidant power": The FRAP assay. Anal. Biochem. 1996, 239, 70-76. 